\title{
I Fórum Latino-Americano de Saúde Mental na Pandemia: desafios, panorama atual e perspectivas futuras
}

\author{
I Latin American Forum of Mental Health in the Pandemic: challenges, current \\ outlook and future prospects
I Foro Latinoamericano de Salud Mental en la Pandemia: desafíos, panorama actual y perspectivas de futuro

Vitor Crestani Calegaro $\mathbb{D}^{a}$, Juliana Motta $\mathbb{D}^{b}$, Mariana Apolinário Fernandes $\mathbb{D}^{c}$, Sigriny Victória Rezer Bertão $\mathbb{D}^{c}$, Heloísa Augusta Castralli $\mathbb{D}^{c}$, Artur Flores Missau $\mathbb{D}^{c}$, Cassiana Cherobini Bortolin $\mathbb{D}^{c}$, Giovanna Fais de Azevedo $\mathbb{D}^{c}$, César Augusto Neumann Ribeiro $\mathbb{D}^{c}$, Natália Gonçalves Rengel $\mathbb{B}^{c}$

a Universidade Federal de Santa Maria, Departamento de Neuropsiquiatria - Santa Maria - Rio Grande do Sul - Brasil; Universidade Federal de Santa Maria, Hospital Universitário de Santa Maria - Santa Maria - Rio Grande do Sul - Brasil; Universidade Federal de Santa Maria, Coordenadoria de Ações Educacionais - Santa Maria - Rio Grande do Sul - Brasil. b Universidade Federal de Santa Maria, Programa de Pós-Graduação em Comunicação - Santa Maria - Rio Grande do Sul - Brasil. ' Universidade Federal de Santa Maria, Curso de Medicina - Santa Maria - Rio Grande do Sul - Brasil.

Instituição: Universidade Federal de Santa Maria - Santa Maria - Rio Grande do Sul - Brasil

DOI 10.5935/2318-0404.20210003

\section{Resumo}

O I Fórum Latino-Americano de Saúde Mental na Pandemia, ocorrido em dezembro de 2020, apresentou 18 estudos desenvolvidos em 13 universidades e instituições do Brasil, Argentina, Chile, Guatemala e Cuba. Os trabalhos foram divididos em cinco mesas: "Correlatos psicológicos da pandemia na população em geral", "Estudos sobre o efeito psicológico e biológico em populações específicas", "Aspectos da pandemia sobre mulheres grávidas e recém-nascidos", "Infância e adolescência na pandemia", e "Terapia e psicologia positiva em tempos de pandemia". O objetivo deste artigo de perspectivas é divulgar as pesquisas expostas e refletir a respeito das principais tendências. Assim, apresenta-se uma síntese de cada trabalho, objetivos e resultados. Os estudos apontam para um aumento nos sintomas de estresse, ansiedade e depressão na população em geral e em grupos específicos como crianças, grávidas, puérperas, profissionais da saúde e imigrantes. Foram sugeridos como possíveis preditores de sofrimento psicológico: exposição excessiva às notícias, insônia, sedentarismo, sexo feminino, jovens, baixa escolaridade, baixa renda, desemprego, abuso emocional ou físico, e diagnóstico 
de transtorno mental. Os estudos apresentados encontram-se em andamento, portanto os dados não são conclusivos. Por fim, as perspectivas e tendências apontadas pelas pesquisas foram discutidas. Em razão da crescente demanda por atendimento psicoterápico, e da escassez de profissionais, principalmente em áreas remotas, o desenvolvimento de novas abordagens de intervenção e promoção da saúde mental são encorajadas.

Palavras-chaves: Saúde mental; Pandemias; Ansiedade; Depressão; Estresse psicológico

\section{Abstract}

The I Latin American Forum on Mental Health in Pandemic, held in December 2020, presented 18 studies developed in 13 universities and institutions in Brazil, Argentina, Chile, Guatemala and Cuba. The studies were divided into five tables: "Psychological correlates of the pandemic in the general population", "Studies on the psychological and biological effect in specific populations", "Aspects of the pandemic on pregnant women and newborns", "Childhood and adolescence in pandemic ", and" Therapy and positive psychology in times of pandemic ". The purpose of this perspective article is to disseminate the research exposed in the forum and reflect about their central trends. Thus, a summary of each work, objectives and results is presented. The studies point to an increase in the symptoms of stress, anxiety and depression in the general population and in specific groups such as children, pregnant women, mothers, health professionals and immigrants. The following factors were suggested as possible predictors of psychological distress: excessive exposure to news, insomnia, physical inactivity, female sex, youth, low education, low income, unemployment, emotional or physical abuse, and diagnosis of mental disorder. The studies presented are ongoing, so the data are not conclusive. Finally, the perspectives and trends pointed out by the surveys were discussed. Due to the growing demand for psychotherapeutic care, and the shortage of professionals, especially in remote areas, the development of new approaches to intervention and promotion of mental health are encouraged.

Keywords: Mental health; Pandemics; Anxiety; Depression; Stress psychological

\section{Resumen}

El I Foro Latinoamericano de Salud Mental en Pandemia, realizado en diciembre de 2020, presentó 18 estudios desarrollados en 13 universidades e instituciones de Brasil, Argentina, Chile, Guatemala y Cuba. Los trabajos se dividieron en cinco tablas: "Correlaciones psicológicas de la pandemia en la población general", "Estudios sobre el efecto psicológico y biológico en poblaciones específicas", "Aspectos de la pandemia en mujeres embarazadas y recién nacidos", "Infancia y adolescencia en pandemia ", y" Terapia y psicología positiva en tiempos de pandemia ". El objetivo de este artículo de perspectivas es difundir las investigaciones expuestas y reflexionar sobre las principales tendencias. Así, se presenta un resumen de cada trabajo, objetivos y resultados. Los estudios apuntan a un aumento de los síntomas de estrés, ansiedad y depresión en la población general y en grupos específicos como niños, mujeres embarazadas, madres, profesionales de la salud e inmigrantes. Se sugirieron los siguientes como posibles predictores de angustia psicológica: exposición excesiva a noticias, insomnio, inactividad física, sexo femenino, juventud, baja educación, bajos ingresos, desempleo, abuso 
emocional o físico y diagnóstico de trastorno mental. Los estudios presentados están en curso, por lo que los datos no son concluyentes. Finalmente, se discutieron las perspectivas y tendencias señaladas por las encuestas. Debido a la creciente demanda de atención psicoterapéutica y la escasez de profesionales, especialmente en áreas remotas, se fomenta el desarrollo de nuevos enfoques de intervención y promoción de la salud mental.

Palabras clave: Salud mental; Pandemias; Ansiedad; Depresión; Estrés psicológico

\section{Introdução}

Mais de um ano após o primeiro caso de COVID-19 (coronavirus disease 2019), registrado em primeiro de dezembro de 2019 em Wuhan (China), a doença ainda é um desafio para profissionais de saúde e pesquisadores. Para evitar a proliferação do vírus, as organizações de saúde passaram a recomendar uma série de orientações de higiene e de convivência, como o distanciamento social e o uso de máscaras. Tanto a doença em si, quanto as medidas adotadas para conter o seu contágio, impactaram no modo de vida das pessoas, que passaram a lidar com a ansiedade e o estresse decorrentes do medo da infecção, mortes de entes queridos, dificuldades financeiras, desemprego, excesso de consumo de informações da mídia, desgaste físico e emocional de trabalhadores da saúde e vulnerabilidade de grupos sociais'. Todos esses elementos, de forma isolada ou em associação, constituem fatores de risco para o desenvolvimento ou piora de diversos transtornos mentais, como transtornos de ansiedade, depressão, transtorno de estresse pós-traumático, uso de substâncias, entre outros².

No intuito de compreender os reais impactos sobre a saúde mental da população no contexto da pandemia de COVID-19, e reduzir as suas consequências, foram iniciados diversos estudos pelo mundo, com predomínio da literatura estrangeira com a população norte-americana, europeia e asiática. Entretanto, o povo latino-americano apresenta as suas particularidades socioculturais, visto que é conhecido por ser festivo, gregário e caloroso, mas também pelas suas marcantes desigualdades econômicas, que são heranças históricas desde a colonização. Assim, o modo de vida e cultura na qual o povo se insere precisa ser levado em consideração no que se refere à generalização dos resultados de estudos estrangeiros. Ademais, ainda antes da pandemia os países da América Latina apresentavam prevalências mais altas de transtornos de ansiedade (com o Brasil no topo do ranking mundial) e depressão do que os países de alta renda ${ }^{3}$.

Nesse contexto, foi realizado o I Fórum Latino-Americano de Saúde Mental na Pandemia (I FLASM), no dia 3 de dezembro de 2020, fruto de uma parceria entre o projeto COVIDPsiq, da Universidade Federal de Santa Maria (UFSM), e o projeto Impacto Emocional por Aislamiento por COVID-19 en Argentina, da Universidad Nacional de Mar Del Plata (Argentina). O evento contou com o apoio da COVIDMINDS Network <https://www. covidminds.org/>, rede de pesquisadores coordenada pela University College London, que possui cadastro de 140 estudos em andamento em todo o mundo. Através dos contatos de pesquisas longitudinais realizadas na América Latina, os coordenadores do evento enviaram convites para todos os pesquisadores cadastrados. Além disso, foram realizados contatos com os responsáveis por pesquisas não cadastradas na plataforma, mas que eram de conhecimento dos pesquisadores. 
O evento ocorreu de forma online e reuniu trabalhos com distintas perspectivas. O fórum teve por objetivo compartilhar resultados preliminares de estudos em andamento, criar redes colaborativas, ampliar a discussão sobre o tema saúde mental e pandemia e, assim, gerar discussões que pudessem contribuir para a elaboração de protocolos de prevenção e tratamentos em saúde mental. Através de transmissão online pelo canal COVIDPsiq no YouTube https://youtu.be/2uy-EB1zViE, com tradução Português-Espanhol, mais de 700 inscritos tiveram a oportunidade de conhecer 18 estudos desenvolvidos em 13 universidades e instituições do Brasil, Argentina, Chile, Guatemala e Cuba. Dois meses após a realização do evento, o vídeo do Fórum já conta com mais de 1.800 visualizações.

Os trabalhos foram agrupados em cinco mesas-redondas: "Correlatos psicológicos da pandemia na população em geral", "Estudos sobre o efeito psicológico e biológico em populações específicas", "Aspectos da pandemia sobre mulheres grávidas e recém-nascidos", "Infância e adolescência na pandemia", e "Terapia e Psicologia Positiva em tempos de pandemia". Este artigo de perspectiva descreve resumidamente os estudos, utilizando a mesma dinâmica de eixos temáticos para apontar as principais contribuições levantadas durante o evento. Assim, são apresentadas, em cada mesa, as respectivas pesquisas, a síntese de cada um dos estudos, os resultados já sistematizados e os pontos de convergência entre eles. Salienta-se que algumas pesquisas ainda estão em andamento, portanto, as referências citam apenas o conteúdo divulgado no Fórum. Por fim, discutese a contribuição dos estudos para a psicoterapia.

\section{Descrição das mesas-redondas}

\subsection{Mesa 1: Correlatos psicológicos da pandemia na população em geral}

A mesa com o tema "Correlatos psicológicos da pandemia na população em geral" foi constituída por quatro pesquisas: "COVIDPsiq: monitoramento da evolução da sintomatologia póstraumática, depressão e ansiedade durante a pandemia de COVID-19 em brasileiros", "COH-FIT: Estudo multinacional colaborativo durante a primeira onda", "Segundas partes nunca son buenas: Miedo a la COVID-19 durante el rebrote em Cuba", e "Impacto emocional de la pandemia en población general: depresión, ansiedad y afecto".

A primeira delas, "COVIDPsiq: monitoramento da evolução da sintomatologia pós-traumática, depressão e ansiedade durante a pandemia de COVID-19 em brasileiros"4, apresentada pelos representantes do grupo de pesquisa COVIDPsiq (Universidade Federal de Santa Maria), Bianca Lorenzi Negretto e Vitor Crestani Calegaro, configurou-se como um acompanhamento sintomatológico da população ao longo da pandemia por COVID-19. Foram apresentados resultados transversais da primeira etapa do estudo, que contou com uma amostra 3625 pessoas, na qual $65 \%$ dos respondentes referiu algum grau de piora em sua saúde mental. Ademais, identificaram-se cinco principais preditores (todos os ps $<0,0001$ ) de sintomas psíquicos: angústia relacionada às notícias, idade (nos mais jovens), diagnóstico psiquiátrico atual, latência do sono e abuso emocional/violência.

Por sua vez, o estudo multinacional "COH-FIT: Estudo multinacional colaborativo durante a primeira onda" ${ }^{5}$ https://www.coh-fit.com/, apresentado no evento pelo pesquisador Felipe Barreto Schuch (Universidade Federal de Santa Maria), além de buscar reconhecer os fatores de risco para desfechos psíquicos negativos, 
dirigiu atenção especial à relação da saúde mental com a preservação da saúde física durante o período pandêmico. $O$ trabalho, que contou com amostra representativa de 1000 participantes em cada um dos dez países avaliados (Brasil, Alemanha, França, Espanha, Estados Unidos, Grécia, Hungria, Itália, Suíça e Inglaterra), ainda não obteve resultados finais. Porém, outro estudo colaborativo realizado anteriormente pelo mesmo grupo pesquisador avaliou cinco países (Brasil, Estados Unidos, Inglaterra, Espanha e Chile), utilizando a mesma amostra representativa de 1000 participantes em cada país, e concluiu que, no Brasil, o tempo gasto com comportamento sedentário aumentou em $40 \%$ e que indivíduos sedentários possuem risco 30\% maior de desenvolver depressão. Ademais, em todos os países participantes, o estudo concluiu que as pessoas mais ativas durante a pandemia têm menor risco de manifestar sintomas de ansiedade e depressão.

Já o estudo "Segundas partes nunca son buenas: Miedo a la COVID-19 durante el rebrote en Cuba", de Yunier Brochè-Pèrez (Universidad Central "Marta Abreu" de Las Villas - Cuba), buscou distinguir fatores associados a sentimentos de medo e percepção de risco, tendo encontrado principalmente: preocupação de contrair o vírus, de outras pessoas ficarem doentes e da volta à vida cotidiana com o vírus ainda em circulação. A pesquisa foi estruturada em duas etapas, com intervalo de 4 meses entre sua realização: na primeira, obtevese como amostra 772 participantes e, na segunda, 373, totalizando 1145 indivíduos. Os resultados encontrados mostraram que o sentimento de medo aumentou ligeiramente na segunda etapa em relação à primeira, visto que passou de $19,04 \%$ para $20,08 \%$ ( $p<0,017$ ). Também se observou que mulheres possuíam maior resposta ao medo em comparação aos homens (3,13 vezes maior para níveis moderados de medo e 3,45 para níveis elevados; $p<0,001$ ). Mediante essa análise, o estudo propõe aprofundar as variáveis preditoras e moduladoras das reações de medo, desenvolver estudos longitudinais que permitam determinar o impacto da pandemia na saúde mental a médio e longo prazo, implementar intervenções orientadas à redução da vulnerabilidade psicológica, e desenvolver alianças entre universidades para a realização de estudos multicêntricos.

Já o trabalho "Impacto emocional de la pandemia en población general: depresión, ansiedad y afecto"7, de Lorena Canet-Juric e Fernando Poo (Universidad Nacional de Mar del Plata), foi um estudo longitudinal dividido em quatro etapas, o qual descreveu e comparou a presença de sintomas de depressão, níveis de ansiedade e afetividade positiva e negativa nas diferentes fases do estudo. Como resultados para ansiedade, o estudo encontrou que: em relação à faixa etária, a sintomatologia ansiosa é maior em indivíduos de 18 e 25 anos, tendendo a aumentar entre os mais idosos e reduzir entre os mais jovens; quanto ao gênero, mulheres apresentaram maiores indicadores de ansiedade do que homens, permanecendo estável durante o tempo; em relação ao nível educacional, quanto maior for, menores eram os sintomas ansiosos. Já os dados encontrados acerca da depressão foram: em relação à faixa etária não apresentaram diferenças, embora tenda a aumentar conforme o decorrer do estudo; em relação ao sexo, mulheres apresentaram maiores indicadores de depressão em comparação aos de homens; quanto ao nível educacional, os sintomas depressivos foram menores em indivíduos com maior nível. Por fim, os resultados acerca da afetividade positiva foram: quanto à faixa etária, idosos apresentaram maiores indicadores quando comparados a indivíduos mais jovens; em relação ao gênero, mulheres apresentaram menor pontuação de afetividade positiva em relação aos homens; por fim, quanto ao nível educacional, pessoas com menor escolaridade apresentaram menores pontuações de afetividade positiva. 
O estudo apontou que os maiores escores de afetividade positiva foram pontuados por idosos, enquanto os menores, por mulheres e indivíduos com menor escolaridade.

Em linhas gerais, compartilhando um caráter vigilante com as manifestações psicoemocionais da sociedade, tais estudos buscaram fornecer um embasamento científico para a elaboração de políticas públicas. Apesar de, no presente momento, nem todas as consequências da pandemia serem completamente medidas e compreendidas, é necessário que a saúde mental seja incorporada às medidas emergenciais de forma global, a fim de tornar possível o planejamento de estratégias de suporte psicológico às pessoas necessitadas, mitigando eventuais efeitos deletérios.

\subsection{Mesa 2: Estudos sobre o efeito psicológico e biológico em populações específicas}

O tema "Estudos sobre o efeito psicológico e biológico em populações específicas" foi discutido em três trabalhos: "Cambios en la salud mental del personal sanitario durante la pandemia de COVID-19 en Argentina: Evidências de un estudio longitudinal", "O impacto do distanciamento social nos ritmos biológicos e na saúde mental: um estudo da efetividade de intervenções em ritmos biológicos e sono", e "Salud Mental de Inmigrantes de Santiago de Chile durante la Pandemia COVID-19".

O primeiro trabalho, intitulado "Cambios en la salud mental del personal sanitario durante la pandemia de COVID-19 en Argentina: Evidências de un estudio longitudinal" ", foi elaborado pelos argentinos Cecilia Lópes Steinmetz e Juan Carlos Godoy (Universidad Nacional de Córdoba e CONICET) em colaboração com Romina Herrera (Guarda de Saúde Mental do Hospital Wenceslao Gallardo). O objetivo desse estudo longitudinal foi examinar as mudanças na saúde mental dos profissionais de saúde durante a pandemia, considerando os principais fatores demográficos da região de residência e outros fatores relacionados à saúde. Os resultados apontaram níveis elevados de ansiedade na população em questão. Entre os 305 profissionais de saúde entrevistados, 56\% expõem níveis elevados de ansiedade na primeira medição; na segunda medição, essa porcentagem aumentou para $66 \%$. Os profissionais de saúde das regiões metropolitanas apresentaram níveis ainda maiores em comparação aos do interior do país na primeira medição, assim como os níveis de ansiedade foram maiores para os profissionais acima de 40 anos. Ademais, houve uma influência significativa entre o tempo e o contágio de COVID-19 em relação à ansiedade. 0 estudo mostrou que os preditores para a mudança no padrão de ansiedade entre a primeira e a segunda medição foram, na ordem decrescente de tamanho de efeito, idade e região versus contágio por coronavírus (não teve versus não sabe, mas teve sintomas).

O segundo trabalho apresentado nesta mesa, "O impacto do distanciamento social nos ritmos biológicos e na saúde mental: um estudo da efetividade de intervenções em ritmos biológicos e sono" ${ }^{\prime \prime}$, de autoria de Luísa K. Pilz e Natividade S. C. Pereira (Universidade Federal do Rio Grande do Sul), e também discutido por Maria Paz Hidalgo, caracteriza-se por observar se a organização circadiana estava associada à saúde durante a pandemia, considerando-se o contexto do distanciamento social, assim como avaliar padrões de atividaderepouso. 0 estudo está em andamento e, até o momento, 175 indivíduos estão participando da análise por 
meio de respostas a questionários e actígrafos. Dentre os participantes, a maioria é jovem, do sexo feminino e com ensino superior completo.

Já o trabalho "Salud Mental de Inmigrantes de Santiago de Chile durante la Pandemia COVID-19"10, de Antonia Errázuriz Concha (Pontificia Universidad Católica de Chile), pretende estimar a prevalência de Episódio Depressivo Grave, a ocorrência de sintomas de depressão e ansiedade na população imigrante, examinar variações em sintomas depressivos e ansiosos nesta população durante e após a pandemia e, por fim, comparar a prevalência de Episódio Depressivo Grave em imigrantes com tais dados da população geral chilena. $O$ estudo é longitudinal, e constitui-se em duas etapas, sendo a primeira realizada em junho de 2020, e a segunda a ser realizada em 2021. A primeira etapa mostrou que a pandemia de COVID-19 gerou aumento na prevalência de indivíduos com depressão moderada e severa, que passou de 14,3\% no período pré-pandemia para $48,9 \%$ durante o isolamento. Da mesma forma, a prevalência de sintomas de ansiedade moderada e severa teve uma prevalência aumentada, passando de $8,2 \%$ no período pré-pandemia para $27,9 \%$ durante o isolamento.

Assim, salienta-se que todos estes trabalhos observaram os efeitos psicológicos oriundos da pandemia de COVID-19 em algumas populações específicas. Nesse aspecto, o aumento dos sintomas de ansiedade e depressão foi uma consequência, frequentemente, relatada nos estudos, tanto em profissionais de saúde argentinos, quanto em imigrantes de Santiago do Chile.

\subsection{Mesa 3: Efeitos da pandemia sobre mulheres gestantes e recém nascidos}

A mesa com o tema "Efeitos da pandemia sobre mulheres gestantes e recém nascidos", abrangeu os seguintes trabalhos: "Coorte de nascidos vivos de Rio Grande 2019: os acompanhamentos WebCovid-19", "Vivências de gestantes e mães de bebês até 12 meses durante a pandemia do coronavírus (COVID-19)", e "Estudio longitudinal sobre el impacto del contexto pandémico sobre la salud mental de mujeres gestantes".

O trabalho "Coorte de nascidos vivos de Rio Grande 2019: os acompanhamentos WebCovid-19"11, de autoria de Christian Loret de Mola Zanatti (Universidade Federal do Rio Grande), é um estudo longitudinal, no qual foram identificados os nascimentos ocorridos nas duas maternidades da cidade de Rio Grande-RS (Hospital Universitário da Universidade Federal do Rio Grande e Santa Casa de Misericórdia de Rio Grande), correspondendo a $99,5 \%$ do total de nascimentos do município. Todas as mães foram convidadas a responder questionários com informações sobre a saúde delas e de seus bebês, objetivando avaliar o efeito da pandemia do COVID-19 e do isolamento social sobre sua saúde geral e mental, ao todo foram 2.052 respondentes incluídas no estudo. A princípio, o acompanhamento seria presencial, contudo, com a pandemia o estudo foi suspenso e reformulado, para ser virtual e focar nos aspectos relacionados ao contexto atual. Assim, foram avaliados três desfechos: depressão (de acordo com a escala EPDS-13), ansiedade (GAD-7) e estresse relacionado à pandemia (Impact of Event Scale). Os resultados demonstraram um aumento das prevalências de depressão e ansiedade com valores maiores que o esperado considerando a prevalência populacional, sendo a maior mudança observada no limiar mais alto de depressão. As mães tiveram uma prevalência de depressão (EPDS13) de cerca de $29 \%$ e de ansiedade grave e moderada de $26 \%$. Em relação ao estresse, mais da metade da 
amostra relatou algum tipo de estresse, mas focando nos níveis moderado e severo a prevalência encontrada foi de $43 \%$.

Já o segundo trabalho apresentado, "Vivências de gestantes e mães de bebês até 12 meses durante a pandemia do coronavírus (COVID-19)"12 <https://momsduringcovid.org/about/>, de Vera Mateus e Ana Osório (Universidade Presbiteriana Mackenzie-SP), faz parte de um estudo internacional financiado pela União Europeia: "Moms During COVID-19". O presente estudo consiste em uma análise longitudinal de 6 meses que visa examinar o impacto da pandemia do COVID-19 na saúde mental perinatal, caracterizando as vivências e formas de lidar com as especificidades das grávidas e mães com bebês de até 6 meses de idade, de modo a identificar potenciais fatores de risco e de proteção. Nesse estudo de 4 etapas (início em 1 de julho de 2020 + 3 momentos de follow-up: $1^{\circ}, 3^{\circ}$ e $6^{\circ}$ mês) foram utilizados o Questionário de Impacto do Coronavírus nas Experiências Perinatais, a Escala de Depressão Pós-natal de Edimburgo (EPDS) e o Questionário de Transtorno de Ansiedade Generalizada (GAD-7), totalizando 918 participantes (280 grávidas e 638 mães com bebês de até 6 meses), destas 471 mulheres aceitaram participar dos momentos de follow-up. Esta queda na adesão também foi observada nos outros países envolvidos no estudo internacional. Foram constatados níveis elevados de ansiedade e depressão durante a pandemia: $44 \%$ das participantes registraram um alto risco de depressão (grávidas - 36\% e pós parto - 47,5\%), e 39\% das participantes apresentaram níveis moderados a severos de sintomas de ansiedade generalizada (grávidas - $34 \%$ e pós parto - $41,7 \%$ ).

Por fim, o "Estudio longitudinal sobre el impacto del contexto pandémico sobre la salud mental de mujeres gestantes" ${ }^{13}$, de Hermán López-Morales (Universidad de Mar del Plata - Argentina), pesquisou longitudinalmente sobre os impactos que a pandemia de COVID-19 causa na saúde de mulheres gestantes na comparação com mulheres não gestantes. No intervalo de 50 dias de isolamento social, a amostra total, composta por 102 mulheres grávidas e um correspondente grupo controle, foi convidada a responder, via formulários virtuais, três questionários: um realizado entre os dias 2 e 5 após a implementação do isolamento social preventivo e obrigatório na Argentina, outro entre os dias 14 e 20 e o último entre os dias 47 e 51 , com o intuito de avaliar principalmente a sintomatologia em depressão e ansiedade e as características sociodemográficas. Os resultados apresentaram um aumento gradual dos indicadores psicopatológicos, embora as mulheres grávidas tenham apresentado um aumento mais acentuado. Nas mulheres gestantes, $32,7 \%$ apresentaram indicadores clínicos de depressão moderada e severa, o que está acima da prevalência esperada de países em desenvolvimento (em torno de 19 e 25\%) e muito acima da prevalência em países desenvolvidos (varia entre 7 e 15\%). Assim, foi detectado maior grau de vulnerabilidade para esse grupo. Em relação à ansiedade, foi observado que grande parte do aumento dos indicadores pode estar relacionada a preocupações com a saúde do feto, com o desenvolvimento da gestação e com o medo de transmissão do vírus de forma vertical. A maior suscetibilidade de gestantes nesse contexto demonstra a necessidade de criação de projetos de políticas públicas, a fim de conseguir um diagnóstico rápido e combater os efeitos da pandemia nessa população específica.

Deste modo, pode-se concluir que estes trabalhos têm em comum a investigação dos impactos da 
pandemia COVID-19 e o isolamento social na saúde perinatal, encontrando maiores níveis de ansiedade e depressão nessas mulheres, o que as torna um grupo vulnerável. Porém, apesar das evidências crescentes do impacto da pandemia na saúde mental, ainda há poucos estudos com essa abordagem e oferta de saúde mental para esse grupo específico.

\subsection{Mesa 4: Infância e adolescência na pandemia}

Na mesa "Infância e adolescência na pandemia", foram expostos três trabalhos: "Rutinas y resultados de salud mental durante las restricciones causadas por COVID-19 em participantes de la Cohorte Chilena de alimentos y médio ambiente (FECHIC)", "Impacto psicológico del aislamento por COVID-19 em niños/as y adolescentes del Gran La Plata", e "¿Y los niños como están? Salud mental en niños argentinos frente a la pandemia por COVID-19".

Abrindo a mesa, o estudo "Rutinas y resultados de salud mental durante las restricciones causadas por COVID-19 em participantes de la Cohorte Chilena de alimentos y médio ambiente (FECHIC)"14, de autoria de Miguel Cordero (Universidad de Chile), descreve a vida em três momentos críticos: gravidez, infância e adolescência. Nessa perspectiva, objetiva-se avaliar, ao longo do período de isolamento, cerca de 3000 indivíduos e, com isso, analisar o impacto do isolamento no comportamento, observando marcadores específicos de estresse, solidão, sintomas de ansiedade e depressão e número total de dificuldades emocionais e comportamentais. A análise foi realizada por meio de questionários virtuais preenchidos por pais e infantes. Assim, até o momento, os resultados obtidos indicaram alterações relevantes no sono (um terço da amostra têm ido dormir perto das 23 horas e começam o dia depois das 9 horas), queda nas interações sociais (um terço da amostra não entrou em contato com amigos na última semana), redução da exposição a atividades escolares (um terço da amostra dedica menos de uma hora por dia para os estudos) e aumento no sentimento de solidão ( $60 \%$ das crianças sentem falta de alguma companhia pela maior parte do tempo).

Em um segundo momento, foi apresentado o trabalho "Impacto psicológico del aislamento por COVID-19 em niños/as y adolescentes del Gran La Plata"15, de autoria de Sandra Marder, Maira Querejeta, Maria Justina Romanazzi e Ana Laguens, juntamente ao Centro de Estudios en Nutrición y Desarrolo Infantil (CEREN) e a Comisión de Investigaciones Científicas de la Provincia de Buenos Aires (CIC). No artigo, analisaram-se dados do questionário sobre as condições de vida e cuidado com a infância durante o isolamento social obrigatório de 150 dias, realizado na região de Gran La Plata. Estão sendo avaliados fatores como hábitos de sono e comportamento. As respostas dos pais indicaram que houve grande impacto no comportamento infantil, como maior frequência em tédio, inquietação, dependência e raiva.

Encerrando a mesa, foi apresentado por Maria Laura Andres (Universidad Nacional de Mar del Plata) o trabalho "¿Y los niños como están? Salud mental en niños argentinos frente a la pandemia por COVID-19"16. O estudo analisa os efeitos da pandemia por COVID-19 em sintomas psicopatológicos em indivíduos de 3 a 18 anos na Argentina, a partir da aplicação de questionários que possibilitaram observar a evolução dos sintomas. Buscou-se, também, verificar se a evolução dos sintomas estava relacionada a fatores como nível 
socioeconômico, grau de isolamento, idade, gênero, entre outros. Em síntese, os resultados indicaram que 45\% dos pais reportaram que as crianças se manifestaram mais irritadiças, entre $40 \%$ a $45 \%$ dos pais consideraram as crianças mais ansiosas e menos ativas, entre $35 \%$ e $40 \%$ observaram que os filhos ficaram mais dependentes, com maiores dificuldades em se concentrar e em dormir, apresentando, inclusive, maiores sintomas de depressão, sendo que as crianças mais novas, significativamente, são as mais afetadas.

Dado o exposto, os artigos convergem na conclusão de que as restrições implementadas em função da pandemia têm especial impacto sobre a qualidade de vida e de desenvolvimento das crianças e adolescentes, sobretudo na qualidade de sono, de aprendizado e no equilíbrio emocional desses indivíduos. Dessa forma, é necessário que sejam pautadas ações para compensar os impactos da pandemia sobre esse grupo específico.

\subsection{Mesa 5: Terapia e psicologia positiva em tempos de pandemia}

A mesa com o tema "Terapia e psicologia positiva em tempos de pandemia" foi constituída por cinco trabalhos: “Atenção em Saúde Mental por Teleatendimento para Profissionais e Alunos de Saúde, Professores e Profissionais de Serviços Essenciais no Contexto da Infecção SARS-CoV-2 - TELEPSI COVID-19", "Levantamento sobre variáveis de Florescimento Humano e Crescimento Psicológico numa Amostra por Conveniência durante a Pandemia pelo Covid-19", "Avaliação da Efetividade das Recomendações de Saúde durante a Pandemia", "La Telepsicología como herramienta para la intervención psicológica en crisis por COVID-19. Experiencias en el contexto cubano", e "Bienestar durante la pandemia de COVID-19".

O primeiro estudo "Atenção em Saúde Mental por Teleatendimento para Profissionais e Alunos de Saúde, Professores e Profissionais de Serviços Essenciais no Contexto da Infecção SARS-CoV-2"17 <https:// telepsi.hcpa.edu.br/>, foi apresentado por Marianna de Abreu Costa (programa TELEPSI do Hospital das Clínicas de Porto Alegre - HCPA). O estudo estruturou um projeto de teleconsulta psicológica e psiquiátrica para profissionais e estudantes da área da saúde, o qual contou com a participação de 2464 registros, sendo $70,3 \%$ profissionais da saúde e 19,2\% professores. Dentre os participantes, 483 foram incluídos em um grupo de prevenção, recebendo, assim, conteúdos de Psicoeducação com e sem vídeos. Ademais, 603 participantes foram selecionados por apresentarem um alto risco para desenvolver sintomas de Ansiedade, Depressão, Irritabilidade ou Burnout, sendo encaminhados para ensaios clínicos de tratamento online: Terapia CognitivoComportamental, Psicoterapia Interpessoal e Psicoeducação com vídeos de suporte. Além disso, o projeto forneceu certificação gratuita para profissionais de saúde que desejaram aplicar seus protocolos. Desse modo, as perspectivas são muito otimistas, tanto pela ajuda psicológica prestada quanto pela quantidade de pessoas que receberam os diversos aconselhamentos.

Ainda sob autoria de Costa, o segundo trabalho apresentado "Levantamento sobre variáveis de Florescimento Humano e Crescimento Psicológico numa Amostra por Conveniência durante a Pandemia pelo Covid-19”18, apresentou um projeto longitudinal, em andamento, sob a perspectiva da Psicologia Positiva. $\mathrm{O}$ estudo tem por objetivo avaliar quais grupos conseguem florescer e se desenvolver durante esse período de estresse que é a pandemia. Dessa forma, o projeto realiza um levantamento, por meio de um formulário 
eletrônico, sobre diversas variáveis positivas de saúde, visando compreender como elas se relacionam com desfechos positivos, e também com desfechos negativos em saúde mental. O projeto encontra-se em fase de coleta de dados, portanto os resultados não foram expostos.

O terceiro trabalho intitulado "Avaliação da Efetividade das Recomendações de Saúde durante a Pandemia"19, apresentado por Maria Paz Loayza Hidalgo (Universidade Federal do Rio Grande do Sul), buscou avaliar a efetividade das recomendações de saúde durante a pandemia e como elas afetam a saúde mental do indivíduo, avaliando a relação dos participantes com o distanciamento, alimentação adequada, atividade física, período de sono e exposição à luz solar. O estudo longitudinal, contou com 409 participantes, cuja maioria era composta por mulheres e jovens, os quais preencheram 3 questionários. Os dados da pesquisa apontaram que $59,66 \%$ dos participantes relataram problemas relacionados ao sono. Ademais, está em progresso uma avaliação de sintomas de humor, ansiedade e estresse dos integrantes da pesquisa. Os resultados serão destinados aos órgãos governamentais para que, caso seja necessário, as recomendações sejam reavaliadas, visando gerar menos efeitos negativos durante o período de isolamento social.

Já a pesquisa "La Telepsicología como herramienta para la intervención psicológica en crisis por COVID-19. Experiencias en el contexto cubano"20, apresentada sob responsabilidade de Evelyn Fernández Castillo (Universidad Central Marta Abreu de Las Villas - Cuba), teve o objetivo de usar a telepsicologia como ferramenta para a atenção psicológica durante a pandemia, identificando as necessidades da população cubana e desenvolvendo ações de ajuda integral para esse grupo. A primeira ação do projeto consistiu em oferecer uma linha de apoio psicológico por telefone e por e-mail, a qual resultou no atendimento direto de mais de 200 pessoas. Outra ação importante foi a criação de 15 boletins de autoajuda que foram baseados na identificação das necessidades da população acerca do novo cenário de pandemia. Esses boletins foram divulgados em redes sociais e, assim, utilizados por mais de 4.000 indivíduos. O projeto também criou grupos de WhatsApp para orientação educativa, difundiu recomendações gerais por meio das redes sociais e o desenvolveu o aplicativo gratuito: "Psicoayuda Vs COVID-19" - que passou a ser compartilhado com o Ministério da Saúde Pública, com o objetivo de propiciar bem-estar durante a pandemia. As principais projeções do estudo são: continuar desenvolvendo estudos sobre as variáveis relacionadas com a saúde mental que permitam compreender melhor o impacto da COVID-19 sobre essas variáveis; fortalecer as ações de Telepsicologia implementadas e estabelecer vínculos com outras instituições e países para o melhor enfrentamento da pandemia.

Encerrando a mesa, o trabalho "Bienestar durante la pandemia de COVID-19"21 das autoras: Ana Alícia Cóbar, Maria de Pilar Grazioce e Marinés Mejía buscou conhecer as necessidades de bemestar e saúde mental da população guatemalteca e realizar intervenção e auxílio psicológico, através de um estudo longitudinal, para desenhar intervenções psicológicas. A investigação foi realizada via um formulário no Google Forms, o qual foi respondido pelos participantes em três etapas com um intervalo de dois meses entre elas. Esse formulário foi divulgado via Whatsapp e e-mail e os dados obtidos foram analisados pelo pacote estatístico SPSS 17.0. Como dados gerais, tem-se que a maioria da amostra foi compreendida por mulheres entre 50 e 60 anos, casadas, latinas, com nível acadêmico universitário e de religião católica. A análise dos dados demonstrou uma correlação 
negativa entre o sexo e as emoções de medo $(r=-0,11)$ e tristeza $(r=-0,11)$, indicando que as mulheres reportaram experimentar essa emoção em sua maioria. Também foram relatadas maiores ocorrências de preocupação e cansaço na população guatemalteca, as quais decorrem, segundo o estudo, do distanciamento de familiares, da situação da economia e da possibilidade de contaminação própria ou de familiar por COVID-19. A partir desses resultados, as intervenções propostas pelo grupo foram psicoterapia e psicoeducação para manejo das emoções dos indivíduos afetados.

Em conclusão, fica explícito que a maioria dos trabalhos apresentados na última mesa possuem um caráter interventivo, posto que todos possuem um propósito comum de trazer melhorias à saúde mental da população - desde a distribuição de materiais informativos e didáticos e até mesmo acompanhamento psicológico - evitando que ocorram agravos maiores. Em conjunto com os resultados obtidos nas apresentações das mesas anteriores, estes trabalhos buscarão trazer, ao longo da pandemia e do momento pós-pandêmico, uma redução de transtornos mentais à população latinoamericana.

Ademais, dois trabalhos versam sobre aspectos positivos da saúde mental, que se manifestam no amadurecimento psicológico e no bem-estar. Estudos sob a perspectiva da Psicologia Positiva fazem um contraponto ao adoecimento psíquico, e devem apresentar em seus resultados, num futuro próximo, características associadas a desfechos favoráveis. O campo da Psicoterapia tem muito a ganhar com essas informações pois podem nortear intervenções para o reforço da resiliência e a promoção da saúde mental.

\section{Discussão}

A partir dos resultados das pesquisas em andamento, é evidente que a pandemia provocou a emergência de sintomas de estresse, ansiedade e depressão na população. As consequências disso, em termos de transtornos mentais, ainda é imprecisa, entretanto, pode-se inferir que existe uma demanda crescente por atendimento nos países latino-americanos. Por ser um povo gregário e caloroso, o distanciamento social pode ser ainda mais doloroso que para povos considerados frios e distantes. Enquanto medidas de contenção de contágio foram adotadas, houve redução da oferta por atendimento psicológico e psiquiátrico. Na ponderação entre a abertura e o fechamento de serviços, deve-se levar em conta fatores relacionados aos pacientes e aos terapeutas. Nesse sentido, as pesquisas sugerem grupos de maior vulnerabilidade aos efeitos da pandemia: crianças, jovens, grávidas, puérperas, profissionais da saúde, e imigrantes. Atenção particular deve ser dada a essas pessoas, a fim de direcionar intervenções de promoção da saúde, prevenção da doença, psicoeducação e tratamento.

Como demonstrado por Broché-Pérez, a segunda onda de contágio retomou sentimentos abrandados pela esperança do fim da pandemia. Nesse mar de incertezas, surge a vacina trazendo a luz em tempos sombrios. Entretanto, a vacinação não ocorre para todos ao mesmo tempo, de forma que esses medos não terão um ponto final a curto prazo. Nesse sentido, a psicoterapia é peça-chave para pacientes ao buscar maior estabilidade emocional interna, enquanto externamente os estressores flutuam conforme esse mar está mais sereno ou mais agitado. 
A exposição excessiva às notícias, insônia, sedentarismo, sexo feminino, jovens, baixa escolaridade, baixa renda, desemprego, abuso emocional ou físico, e o diagnóstico de transtorno mental foram apontados pelas pesquisas apresentadas no fórum como possíveis preditores para uma sintomatologia elevada. São fatores que influenciam a prática da psicoterapia, seja no que se refere ao acesso dos pacientes, seja na sua condução, seja no foco das intervenções. O medo pandêmico tornou-se um sentimento comum e persistente, que pode ser direta ou indiretamente ligado à COVID-19: de ser infectado, de transmitir para outras pessoas, de sentir-se sozinho, de sofrer abusos, de perder o emprego, de não conseguir pagar as dívidas, etc ${ }^{22}$. Mesmo a negação da pandemia traz o medo em seu íntimo, nas suas formas mais primitivas, exteriorizado de forma agressiva: de não conviver, de perder oportunidades, de ter que aceitar o inaceitável. A raiva, a culpa, o martírio, a dúvida e a desconfiança permeiam as relações interpessoais e se inserem na setting, trazendo desafios que paciente e terapeuta necessitam trabalhar. $O$ resultado pode ser muito positivo e enriquecedor, mas também pode inviabilizar o tratamento. Um exemplo disso é quando o paciente necessita urgentemente de uma consulta e o terapeuta está em isolamento devido ao contato com uma pessoa com COVID-19, trazendo a emergência de sentimentos de desamparo. Outro exemplo é quando o paciente relata no final da sessão, de forma inconsequente, que teve contato com uma pessoa infectada, e esse fato gera sentimentos negativos no terapeuta. Nesse contexto, a modalidade de atendimento, bem como o foco da psicoterapia, deve ser reavaliada, e mudanças no setting podem ser necessárias. A alternativa online é uma opção a ser considerada, mas se deve ter em conta os benefícios e prejuízos da técnica, que não serão discutidos neste artigo.

A mesa 5, "Terapia e psicologia positiva em tempos de pandemia", apresentou pesquisas que investigam e desenvolvem o uso de intervenções. A Telepsicoterapia vem possibilitando a manutenção dos atendimentos e o acolhimento de pessoas em sofrimento mental decorrente da pandemia e de seus desdobramentos. Dessa forma, tornou-se uma ferramenta imprescindível. Na pandemia, as convicções de um setting ideal de atendimento impossível deram lugar ao possível e real. Os benefícios ao utilizar a Telepsicoterapia no que tange à superação dos limites impostos pelas barreiras físicas foram aprendidos por pacientes e terapeutas, de forma que não é possível mais retroceder esse passo. Por exemplo, o oferecimento de atendimento, consultoria e supervisão para pessoas em áreas remotas é um grande ganho que pode ser ainda mais explorado. Por outro lado, pós-pandemia a técnica não deve ser vista como substitutiva da terapia presencial, motivada pelo entusiasmo causado pela maior aceitação desta modalidade, com fins outros que não o benefício dos pacientes.

Para além do setting e das terapias habituais, mesmo online, a demanda crescente por atendimento em saúde mental estimula terapeutas e pesquisadores a buscarem novas possibilidades de intervenção. Assim como nem todo o estresse é traumático, e em certa medida, proporciona o amadurecimento da personalidade, é preciso olhar para a sociedade buscando o saudável ${ }^{23,24,25}$. Nesse sentido, é possível aprender com as experiências das pessoas, seja no consultório, seja na vida, para compreender que os caminhos da resiliência são múltiplos e inerentes à cultura, à sociedade e à biologia. Dessa forma, a mesa 5 apresentada no fórum apontou para uma tendência ao enfoque dos aspectos saudáveis do Ego, que podem ser grandes aliados terapêuticos, uma vez que resultam na ampliação das possibilidades de intervenção. Dado que a carência de cuidados em saúde mental, que já era uma realidade antes da pandemia, tende a tornar-se ainda maior após. 
Neste cenário, práticas e técnicas que buscam um estabelecimento de equilíbrio, como atividades físicas, promoção do sono, resolução de problemas, meditação, mindfullness, aceitação e gratidão, encontram em meio terreno fértil para desenvolvimento.

O presente artigo possui limitações quanto à síntese de diversos trabalhos que ainda estão em andamento. Ressalta-se que os autores de cada estudo apresentado no I FLASM tiveram autonomia para expor da melhor maneira possível aquilo que tinham até o momento do evento. Dessa forma, não houve um padrão na exposição dos resultados estatísticos, e os trabalhos apresentaram dados incompletos. Portanto, as informações apresentadas neste artigo não podem ainda ser generalizadas, e deve-se aguardar a finalização e publicação dos estudos para maiores conclusões. Apesar dessas limitações, os autores trazem um panorama geral acerca das pesquisas que abordam a temática da saúde mental durante a pandemia de COVID-19 no cenário da América-Latina e apontam para direções futuras no campo da psicoterapia e das intervenções possíveis neste contexto.

\section{Conclusão}

O I Fórum Latino-Americano de Saúde Mental na Pandemia foi um marco de colaboração entre pesquisadores da saúde mental no contexto da pandemia. O evento agrupou 13 pesquisas realizadas por 18 universidades em cinco mesas-redondas, incluindo dados coletados a partir da população geral, populações específicas, grávidas, recém nascidos, infância e adolescência; e estudos sobre terapia e Psicologia Positiva. Os resultados preliminares apresentados demonstraram um aumento nos sintomas de estresse, ansiedade e depressão na população em geral, e especificamente, em crianças, grávidas, puérperas, profissionais da saúde e imigrantes. Ao final das apresentações de cada mesa, os pesquisadores levantaram os pontos mais relevantes dos estudos, destacando a importância do tema na atualidade, bem como o impacto que a pandemia e o isolamento social causaram na saúde mental da população latino-americana.

As pesquisas apresentaram diversos pontos de convergência e, apesar de mostrarem dados preliminares, apontaram para a necessidade de medidas de saúde pública para reduzir os impactos negativos da pandemia na saúde mental. Especificamente, os fatores estudados impactam diretamente na prática da psicoterapia. Psicoterapeutas devem buscar uma profunda compreensão sobre o momento atual, a fim de entender os efeitos da pandemia para paciente, terapeuta e setting. Finalmente, o desenvolvimento de novas abordagens de intervenção e promoção da saúde mental são encorajadas. Sugere-se a manutenção do diálogo entre os pesquisadores de diferentes países para a complementação dos resultados e para validação dos mesmos.

\section{Agradecimentos}

Agradecimentos especiais à Dra. Lorena Canet Juric (Universidad Nacional de Mar del Plata), pela colaboração na organização do evento. Também agradecemos ao apoio da COVIDMINDS Network, através da Dra. Alexandra Bradbury (University College London), e da Coordenadoria de Ações Educacionais, representada pela Dra. Silvia Maria de Oliveira Pavão (UFSM). 


\section{Referências}

1. J.M. Cénat, R.D. Dalexis, M. Guerrier, P.G. Noorishad, D. Derivois, J. Bukaka, J.P. Birangui, K. Adansikou, L.A. Clorméus, C.K. Kokou-Kpolou, A. Ndengeyingoma, V. Sezibera, R.E. Auguste, C. Rousseau, (2021). Frequency and correlates of anxiety symptoms during the COVID-19 pandemic in low- and middle-income countries: a multinational study. J. Psychiatr. Res., 132, pp. 13-17, 10.1016/j.jpsychires.2020.09.031.

2. Cao, W., Fang, Z., Hou, G., Han, M., Xu, X., Dong, J., Zheng, J., (2020). The psychological impact of the COVID-19 epidemic on college students in China. DOI: 10.1016/j. psychres.2020.112934.

3. Global Burden of Disease Collaborative Network. Global Burden of Disease Study 2019 (GBD 2019) Results. Seattle, United States: Institute for Health Metrics and Evaluation (IHME), 2020. Disponível em: <http:// ghdx.healthdata.org/gbd-results-tool>. Acesso em: 19 de março de 2021.

4. Calegaro VC, Negretto, BL. COVIDPsiq: monitoramento da evolução da sintomatologia póstraumática, depressão e ansiedade durante a pandemia de COVID-19 em brasileiros. In: 10 Fórum Latino-Americano de Saúde Mental na Pandemia; 2020 Dez 3; Santa Maria, BR.

5. Schuch F. COH-FIT: Estudo multinacional colaborativo durante a primeira onda. In: 10 Fórum LatinoAmericano de Saúde Mental na Pandemia; 2020 Dez 3; Santa Maria, BR.

6. Broche-Pérez Y. Segundas partes nunca son buenas: Miedo a la COVID-19 durante el rebrote en Cuba. In: 10 Fórum Latino-Americano de Saúde Mental na Pandemia; 2020 Dez 3; Santa Maria, BR.

7. Canet-Juric L, Poo F. Impacto emocional de la pandemia en población general: depresión, ansiedad y afecto. In: 1ㅇ Fórum Latino-Americano de Saúde Mental na Pandemia; 2020 Dez 3; Santa Maria, BR.

8. Steinmetz LCL, Godoy JC, Herrera, R. Cambios en la salud mental del personal sanitario durante la pandemia de COVID-19 en Argentina: Evidências de un estudio longitudinal. In: 10 Fórum Latino-Americano de Saúde Mental na Pandemia; 2020 Dez 3; Santa Maria, BR.

9. Pilz LK, Pereira NSC, Hidalgo MP. O impacto do distanciamento social nos ritmos biológicos e na saúde mental: um estudo da efetividade de intervenções em ritmos biológicos e sono. In: 10 Fórum Latino-Americano de Saúde Mental na Pandemia; 2020 Dez 3; Santa Maria, BRConcha AE.

10. Concha AE. Salud Mental de Inmigrantes de Santiago de Chile durante la Pandemia COVID-19. In: 10 Fórum Latino-Americano de Saúde Mental na Pandemia; 2020 Dez 3; Santa Maria, BR.

11. Zanatti CLM. Coorte de nascidos vivos de Rio Grande 2019: os acompanhamentos WebCovid-19. In: 10 Fórum Latino-Americano de Saúde Mental na Pandemia; 2020 Dez 3; Santa Maria, BR.

12. Mateus V, Osório A. Vivências de gestantes e mães de bebês até 12 meses durante a pandemia do coronavírus (COVID-19). In: 1ㅇ Fórum Latino-Americano de Saúde Mental na Pandemia; 2020 Dez 3; Santa Maria, BR.

13. López-Morales H. Estudio longitudinal sobre el impacto del contexto pandémico sobre la salud mental de mujeres gestantes. In: 1o Fórum Latino-Americano de Saúde Mental na Pandemia; 2020 Dez 3; Santa Maria, BR.

14. Cordero M. Rutinas y resultados de salud mental durante las restricciones causadas por COVID-19 em participantes de la Cohorte Chilena de alimentos y médio ambiente (FECHIC). In: 10 Fórum Latino-Americano de Saúde Mental na Pandemia; 2020 Dez 3; Santa Maria, BR.

15. Marder S. Impacto psicológico del aislamento por COVID-19 em niños/as y adolescentes del Gran La Plata. In: 1ํ Fórum Latino-Americano de Saúde Mental na Pandemia; 2020 Dez 3; Santa Maria, BR.

16. Andres ML. ¿Y los niños como están? Salud mental en niños argentinos frente a la pandemia por COVID-19. In: 10 Fórum Latino-Americano de Saúde Mental na Pandemia; 2020 Dez 3; Santa Maria, BR.

17. Costa MA. Atenção em Saúde Mental por Teleatendimento para Profissionais e Alunos de Saúde, Professores 
e Profissionais de Serviços Essenciais no Contexto da Infecção pela SARS-CoV-2. In: 10 Fórum LatinoAmericano de Saúde Mental na Pandemia; 2020 Dez 3; Santa Maria, BR.

18. Costa MA, Manfro GG. Levantamento sobre variáveis de Florescimento Humano e Crescimento Psicológico numa Amostra por Conveniência durante a Pandemia pelo Covid-19. In: 1ํ Fórum atino-Americano de Saúde Mental na Pandemia; 2020 Dez 3; Santa Maria, BR.

19. Hidalgo MP. Avaliação da Efetividade das Recomendações de Saúde durante a Pandemia. In: 1ㅇo Fórum Latino-Americano de Saúde Mental na Pandemia; 2020 Dez 3; Santa Maria, BR.

20. Castillo EF. La Telepsicología como herramienta para la intervención psicológica en crisis por COVID-19. Experiencias en el contexto cubano. In: 10 Fórum Latino-Americano de Saúde Mental na Pandemia; 2020 Dez 3; Santa Maria, BR.

21. Cóbar AA, Grazioce MP, Mejía M. Bienestar durante la pandemia de COVID-19. In: 10 Fórum LatinoAmericano de Saúde Mental na Pandemia; 2020 Dez 3; Santa Maria, BR.

22. Ornell, F., Schuch, J.B., Sordi, A.O., Kessler, F.H.P., (2020). "Pandemic fear" and COVID-19: mental health burden and strategies. Rev. Bras. Psiquiatr. 42, 232-235. https://doi.org/10.1590/1516-4446-2020-0008

23. Bonanno, G.A., (2004). Loss, Trauma, and Human Resilience: Have We Underestimated the Human Capacity to Thrive after Extremely Aversive Events? Am. Psychol. 59, 20-28. https://doi.org/10.1037/0003066X.59.1.20

24. Calegaro, V.C., Mosele, P.H.C., Negretto, B.L., Zatti, C., da Cunha, A.B.M., Freitas, L.H.M., (2019). The role of personality in posttraumatic stress disorder, trait resilience, and quality of life in people exposed to the Kiss nightclub fire. PLoS One 14, e0220472. https://doi.org/10.1371/journal.pone.0220472

25. Jakšć, N., Brajković, L., Ivezić, E., Topić, R., Jakovljevíc, M., Jakšić, N., Brajković, L., Ivezić, E., Topić, R., Jakovljević, M., (2012). The role of personality traits in posttraumatic stress disorder (PTSD). Psychiatr. Danub. 24, 256-66.

Contribuições: Vitor Crestani Calegaro -Conceitualização, Gerenciamento do Projeto, Redação - Preparação do original, Redação - Revisão e Edição, Supervisão;

Juliana Motta - Redação - Preparação do original, Redação - Revisão e Edição, Supervisão;

Mariana Apolinário Fernandes - Redação - Preparação do original, Redação - Revisão e Edição

Sigriny Victória Rezer Bertão - Redação - Preparação do original, Redação - Revisão e Edição;

Heloísa Augusta Castralli - Redação - Preparação do original, Redação - Revisão e Edição;

Artur Flores Missau - Redação - Preparação do original, Redação - Revisão e Edição;

Cassiana Cherobini Bortolin - Redação - Preparação do original, Redação - Revisão e Edição;

César Augusto Neumann Ribeiro - Redação - Preparação do original, Redação - Revisão e Edição;

Giovanna Fais de Azevedo - Redação - Preparação do original, Redação - Revisão e Edição;

Natália Gonçalves Rengel - Redação - Preparação do original, Redação - Revisão e Edição.

\section{Correpondência}

Vitor Crestani Calegaro

vitor.calegaro@ufsm.br 
Submetido em: 12/02/2021

Aceito em: 14/04/2021 